\title{
Empty follicle syndrome after GnRH agonist trigger, rescued with HCG in a donor oocyte with successful pregnancy outcome: a case report
}

\author{
Akshaya Kumar Mahapatro*, Kundavi Shankar, Thankam Varma
}

Institute of Reproductive Medicine, Madras Medical Mission, Chennai, Tamil Nadu, India

Received: 04 December 2015

Accepted: 07 January 2016

\author{
*Correspondence: \\ Dr. Akshaya Kumar Mahapatro, \\ E-mail: dr.aks73@gmail.com
}

Copyright: () the author(s), publisher and licensee Medip Academy. This is an open-access article distributed under the terms of the Creative Commons Attribution Non-Commercial License, which permits unrestricted non-commercial use, distribution, and reproduction in any medium, provided the original work is properly cited.

\begin{abstract}
Empty follicle syndrome (EFS) is a rare condition characterized by failure to obtain oocytes despite repeated meticulous aspiration from normally growing ovarian follicles during in vitro fertilization (IVF) cycles. Here we report a case of empty follicle syndrome in donor oocyte cycle after gonadotropin releasing hormone agonist (GnRHa) triggering for final oocyte maturation. Her estradiol on the day of trigger was 4564.3 IU/L. No oocytes were collected from the right ovary and the procedure was abandoned. The patient was successfully rescued by retriggering with 10,000 units of Inj. hCG and 5 oocytes were collected after 35 hours of retriggering. All oocytes were mature (MII) and fertilized by recipient's husband sperm. Recipient cycle was prepared by hormone therapy, and underwent day 3 fresh embryo transfer. Successful pregnancy was achieved and a term female child was delivered at 37 weeks by lower segment caesarean section. Management options of EFS in hyper responder patients triggered with GnRH agonist may be a risk factor for empty follicle syndrome \& retriggering with Rescue hCG might help in oocyte retrieval in a donor.
\end{abstract}

Keywords: Empty follicle syndrome, GnRH agonist, Ovarian hyperstimulation syndrome, HCG, Oocyte pick up

\section{INTRODUCTION}

Failure to aspirate oocytes from follicles with meticulous follicular aspiration after Controlled ovarian stimulation $(\mathrm{COH})$ in a patient having apparently normal growth of follicles and steroidogenesis is called empty follicular syndrome. It is a frustrating complication of ART. This could be traumatic for both the couple \& the treating clinician. EFS was first reported by Coulam et al in 1986. ${ }^{1}$

Later it has been classified into 'genuine' and 'false' types by Stevenson and Lashen. ${ }^{2}$ Genuine type has been defined as a failure to retrieve oocytes despite optimal hCG levels on the day of oocyte retrieval. Whereas false type has been defined as a failure to retrieve oocytes in the presence of low hCG due to an error in the administration or poor bioavailability of hCG. ${ }^{2,3}$ The reported prevalence of EFS is $0.045-7 \%$ and is mostly FEFS. ${ }^{4}$ When hCG was used as triggering agent different studies suggest that minimum serum $\beta$ hCG concentration ranges from5 to $161 \mathrm{IU} / \mathrm{l}^{2,5}$

Ovarian hyperstimulation syndrome (OHSS) is a potentially life threatening condition and is the most serious iatrogenic complication of controlled ovarian hyperstimulation cycles. ${ }^{6}$ A single dose of GnRH agonist (GnRHa) for final oocyte maturation has been proposed as an effective strategy to prevent OHSS and as a first line protocol for treatment of oocyte donors. ${ }^{7}$ Recently, however few case-reports described empty follicle syndrome (EFS) after GnRHa triggering in OHSS risk patients, despite the presence of a large number of apparently mature follicles at the oocyte pick-up (OPU). ${ }^{8}$ FEFS is commonly described to a patient error in timing, preparation or administration of the triggering drug or to problem with its manufacturing, packing or shelf life. ${ }^{9,10}$ 
Ovulation triggering, with its effect on loosening the cumulus oocyte complex from the follicle wall play a role in pathophysiology of EFS. There is only one study which reported prevalence of EFS comparing HCG versus GnRH agonist as trigger. ${ }^{11}$

\section{CASE REPORT}

27 yrs female known hypothyroidism on Eltroxine 75 mcg for last 1 year with primary subfertility for 2 yrs with regular menstrual cycle, right hydrosalpinx \& left patent fallopian tube on HSG who failed to conceive after 4 cycle of ovulation induction (OI) \& timed intercourse presented to IRM OPD for treatment. On evaluation the male partner was $32 \mathrm{yrs}$ old having normal clinical finding $\&$ normal semen analysis result. Female partner had BMI $22.68 \mathrm{~kg} / \mathrm{m} 2$, clinical examination revealed no abnormality. On evaluation patient was diagnosed as a case of diminished ovarian reserve due to low antral follicle count (RO AFC-3/ LO-1) \& low serum AMH $(0.43 \mathrm{ng} / \mathrm{dl})$ with normal female karyotype $(46 \mathrm{XX})$. Patient was counselled about diminished ovarian reserve $\&$ therapeutic trial with antagonist protocol was planned. Controlled ovarian stimulation was started with recombinant FSH (Recagon- 350 units /day.). After 5 days of gonadotrophin stimulation the response was sub optimal, Inj. cetrorelix $0.25 \mathrm{mg}$ was started from day 6 of gonadotrophins. As per patients request she was stimulated for another four days with inj Recagon 350 units \& Inj Menopur 112.5 units (HMG). As she produced only 2 dominant follicles, IUI-H was planned but the outcome was negative. Patient was counselled for donor oocyte programme in view of Low AFC, low $\mathrm{AMH}$ level \& borderline elevated FSH.

\section{Prospective donor}

25 yrs para -2 living-2 abortion 1 not sterilised with both normal vaginal delivery was selected as a prospective egg donor. On evaluation her menstrual cycle was regular, with nil significant past \& family history. Her BMI was $25.29 \mathrm{~kg} / \mathrm{m} 2$ \& clinical examination revealed no abnormality. On investigation, screening was negative, Day-2 serum FSH, LH, E2, progesterone, prolactin \& thyroid profile was with in normal limits, \& antral follicle count was 16 (ROAFC-10/ LO-6). Antagonist protocol was used. Controlled ovarian stimulation started with recombinant FSH 225 units (inj Folligraf) \& stimulated for nine days. Inj cetrorelix $0.25 \mathrm{mg}$ (GnRH antagonist) was started from day 6 of gonadotrophins. On the day of trigger her serum estradiol (E2) was 4564.3 \& LH -0.931. Ovulation was triggered with inj Triptorelin $200 \mathrm{mcg}$ in view of high E2 level (risk for severe ovarian hyper stimulation syndrome) when 3 follicles had a size of $>18$ $\mathrm{mm}$ diameter. Oocyte retrieval was done after $35 \mathrm{hrs}$ of GnRH agonist trigger. . The procedure of egg collection was abandoned after aspiration of 7 good follicles as no eggs were collected. Serum $\beta$ hcg was not done as she was triggered with agonist \& rescue inj HCG 10000 was given immediately. Serum $\beta$ hcg was $87.91 \mathrm{mIU} / \mathrm{ml}$ before oocyte retrieval. Oocytes retrieval was done after 35 hours of trigger and 5 M2 oocytes collected. ICSI was done by using recipient's husband sperm. All 5 oocytes were fertilized \& three cleaved. Recipient was prepared by hormone therapy for embryo transfer $\&$ on day 3 , fresh embryo transfer was done (3X8cell). Post embryo transfer she was treated with luteal support. Serum $\beta$ hcG level on day 16 of ET was $862.55 \mathrm{miu} / 1$.Pregnancy was confirmed as single intrauterine live fetus by ultrasonography. She underwent emergency lower segment caesarean section at 37 weeks due to type 2 anterior placenta praevia \& delivered a healthy term female child weighing $3 \mathrm{~kg}$.

\section{DISCUSSION}

The incidence of EFS has been reported as $0.6-3.5 \%$ in $\mathrm{GnRH}$ agonist trigger cycles, which is similar to that reported (0.1-3.1\%) after an hCG trigger in all patient groups including hypo-normo and hyperresponders. ${ }^{11}$ EFS is a rare and frustrating complication of IVF. As described previously, the exact etiology of EFS after hCG triggering is not fully explained. A systematic review reported that $67 \%$ of cases were so called false empty follicle syndrome mainly related to human error or pharmacological problems. ${ }^{2}$

Two classes of EFS can be differentiated by hormone levels on the day of ovum pick up. In genuine EFS the level of either HCG, LH or progesterone are concordant with the correct administration of triggering drug while false EFS the levels are low. When hcg was used as triggering agent different studies suggest that minimum serum $\beta$ hcg concentration ranges from 5 to $161 \mathrm{IU} / \mathrm{l}^{2,5} \mathrm{In}$ case of ovulation trigger with GnRH agonist, no cut off levels of LH or progesterone on the day of ovum pick up has been proposed. In contrast to hCG triggering, the action of GnRHa trigger is indirect via the endogenous release of LH and FSH from the pituitary. ${ }^{12,13}$

In a retrospective study by Kummer et al 8 to 12 hours after triggering all the cases of EFS had an LH level of 15 $\mathrm{IU} / \mathrm{l}$, and a progesterone level of $3.5 \mathrm{ng} / \mathrm{ml} .^{14}$

HCG performs its action directly on the ovary; EFS may originate in folliculogenesis or in the ovarian response to the triggering stimulus. In GnRHa, the fault may include an inability of the pituitary to release LH to trigger ovulation, or a failure in one of the mediators/receptors on the ovary. Borderline hypothalamic -pituitary dysfunction may be an etiology for EFS after GnRH agonist trigger. ${ }^{11,14}$ Management of patients with EFS is a challenge for clinicians. Unfortunately no single treatment is known to be universally effective. ${ }^{9}$ The most important preventive intervention should be the confirmation of the correct application of the triggering agent by the healthcare professional or the patient. In our case we confirmed the injection was given properly. Nevertheless we can't be sure about the other potential factors related with false EFS such as manufacturer 
defects and low bioavailability. Retriggering with a higher dose of $\mathrm{GnRh}$ agonist might be another solution in EFS cases after GnRH agonist triggering. Rescue with hCG might be a better option in EFS cases after agonist triggering when the exact mechanism is unclear. The retrospective study by AnnBlazquez et al showed that ovum pick up rate in a rescue protocol with a second triggering agent by Inj HCG is more effective than retriggering with $\mathrm{GnRH}$ agonist. ${ }^{16}$ Another point in these cases is the dose of hCG used for retriggering, higher dose might be risky in a setting where cryopreservation was not available. In our case as the patient was a donor, triggered with 10000 units of HCG and after oocytes retrieval, she was monitored for OHSS and prophylaxis treatment given.

\section{CONCLUSION}

To conclude, Retriggering with HCG \& freezing of embryos might be a rescue protocol in management of EFS in OHSS prone patients triggered with GnRH agonist.

Funding: No funding sources Conflict of interest: None declared

Ethical approval: Not required

\section{REFERENCES}

1. Coulam CB, Bustillo M, Schulman JD. Empty follicle syndrome. Fertil steril. 1986;46:1153-5.

2. Stevenson TL, Lashen H. Empty follicle syn $\neg$ drome: the reality of a controversial syndrome, a systematic review. Fertil Steril. 2008;90:691-8.

3. Kim JH, Jee BC. Empty follicle syndrome. Clin Exp Reprod Med. 2012;39(4):132-7.

4. Mesen TB, Yu B, Richter KS, Widra E, DeCherney $\mathrm{AH}$, Segars JH. The prevalence of genuine empty follicle syndrome. Fertil Steril. 2011;96:1375-57.

5. Reichman DE, Greenwood E, Meyer L, Kligman I, Rosenwaks Z. Can in vitrofertilization cycles be salvaged by repeat administration of intramuscular human chorionic gonadotropin the day after failed injection? Fertil Steril. 2012;98:671-4.

6. Humaidan P, Kol S, Papanikolaou E. The Copenhagen GnRH Agonist Triggering Workshop Group. GnRH agonist for triggering of final oocyte maturation: time for a change of practice? Hum Reprod Update. 2011;17:510-4.
7. Humaidan P, Quartarolo J, Papanikolaou EG. Preventing ovarian hyperstimulation syndrome: guidance for the clinician. Fertil Steril. 2010;94(2):389-400.

8. Honnma H, Hashiba Y, Asada Y, Endo T. Failure of triggeringoocyte maturation with a GnRH agonist in polycystic ovary syndrome: two case reports. Eur J Obstet Gynecol Reprod Biol. 2011;157(2):239-40.

9. Reichman DE, Hornstein MD, Jackson KV, Racowsky C. Empty follicle syndrome does repeat administration of hCG really work? Fertil Steril. 2010;94:375-77.

10. Zegers-Hochschild F, Fernandez E, Mackenna A, Fabres C, Altieri E, Lopez T. The empty follicle syndrome: a pharmaceutical industry syndrome. Hum Reprod. 1995; 10:2262-5.

11. Castillo JC, Garcia-Velasco J, Humaidan P. Empty follicle syndrome after GnRHa triggering versus hCG triggering in COS. J Assist Reprod Genet. 2012;29:249-53.

12. Gonen Y, Balakier H, Powell W, Casper RF. Use of gonadotropin releasing hormone agonist to trigger follicular maturation for in vitro fertilization. J Clin Endocrinol Metab. 1990;71:918-22.

13. Itskovitz J, Boldes R, Levron J, Erlik Y, Kahana L, Brandes JM. Induction of preovulatory luteinizing hormone surge and prevention of ovarian hyperstimulation syndrome by gonadotropin releasing hormone agonist. Fertil Steril. 1991;56:213-20.

14. Kummer NE, Feinn RS, Griffin DW, Nulsen JC, Benadiva CA, Engmann LL. Predicting successful induction of oocyte maturation after gonadotropin releasing hormone agonist (GnRHa) trigger. Hum Reprod. 2013;28:152-9.

15. Quintans CJ, Donaldson MJ, Blanco LA, Pasqualini RS. Empty follicle syndrome due to human errors: its occurrence in an in-vitro fertilization programme. Hum Reprod. 1998;13:2703-5.

16. Anna Blazquez, Juan Jose' Guille'n, Clara Colome', Oriol Coll, Rita Vassena, Valerie Vernaeve. Empty follicle syndrome prevalence and management in oocyte donors. Human Reproduction. 2014;29:22217.

Cite this article as: Mahapatro AK, Shankar K, Varma T. Empty follicle syndrome after GnRH agonist trigger, rescued with HCG in a donor oocyte with successful pregnancy outcome: a case report. Int J Reprod Contracept Obstet Gynecol 2016;5:549-51. 